\title{
Investigation of Dynamic Thiol Disulfide Homeostasis in Acute Respiratory Failure Patients in Intensive Care Unit
}

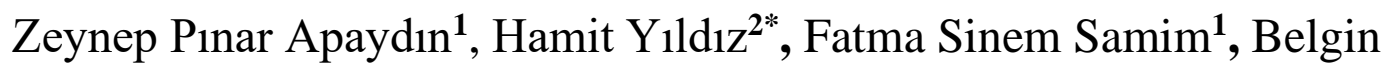 \\ Alaşehirli $^{1}$
}

${ }^{1}$ Gaziantep University, Faculty of Medicine, Department of Pharmacology, Gaziantep, Turkey.

${ }^{2}$ Gaziantep University, Faculty of Medicine, Department of Internal Medicine, Gaziantep, Turkey.

\begin{abstract}
Thiols are antioxidant agents which belong to mercaptan group consisting of sulfur and carbon containing sulfhydryl (-SH). Thiol levels, in the case of elevated oxidative stress, are lowered by their use in neutralization of reactive oxygen molecules. It was aimed to investigate thiol and disulfide blood levels or thiol/disulfide ratio as a marker of total oxidant status which plays a role in the pathogenesis of inflammatory diseases that may cause respiratory failure. The study included 98 patients (58 males, 40 females) who have partial oxygen pressure $(\mathrm{PaO} 2)<60 \mathrm{mmHg}$ or partial carbon dioxide pressure $(\mathrm{PaCO} 2)>45 \mathrm{mmHg}$ in arterial blood in intensive care unit and 98 healthy volunteers. Total thiol and native thiol levels were measured by spectrophotometric method. Total thiol (270 \pm 99.81$)$, native thiol $(203.90 \pm 103.41)$ and disulfide $(33.10 \pm 12.42)$ levels of the patient group were significantly lower $(p<0.001)$ than total thiol (423.62 \pm 70.3$)$, native thiol (307.13 \pm 57.73$)$ and disulfide $(58.24 \pm 27.21)$ of the control group levels. There is no significant difference between native thiol / total thiol, disulfide / total thiol and disulfide / native thiol ratios. This study may be the first study in the literature in terms of providing the diagnosis and follow-up of dynamic thioldisulfide balance in patients with acute respiratory failure requiring intensive care support treatment. Thiol and disulfide blood levels or thiol / disulfide ratio can guide us as a prognostic test in acute respiratory failure patients.
\end{abstract}

Key words: Native thiol, Oxidative stress, Respiratory failure, Thiol/disulfide homeostasis,

Total thiol.

\section{Introduction}

Acute respiratory failure is a syndrome characterized by a sudden deterioration in the ability of the respiratory system to maintain adequate gas exchange, thus not providing enough oxygen $\left(\mathrm{O}_{2}\right)$ to meet the needs of the tissues and /or not eliminating the metabolism product carbon dioxide $\left(\mathrm{CO}_{2}\right)$. It can occur in a healthy person for a cause such as pneumonia or as an acute

\footnotetext{
* Corresponding Author: Hamit Y1ldı, Tel: +90 544908 9090, E-mail: drhyildiz@ @otmail.com, ORCID ID: 0000-0001-7858-5123.
} 
exacerbation in patients with chronic respiratory failure. Since acute respiratory failure is a clinical condition that can develop within minutes or hours and cause vital changes in blood gases and acid-base balance, early diagnosis and treatment are important in terms of the survival of the patient. Diagnosis of acute respiratory failure is made if the partial arterial oxygen pressure $\left(\mathrm{PaO}_{2}\right)$ is below $60 \mathrm{mmHg}$ or the partial arterial carbon dioxide pressure $\left(\mathrm{PaCO}_{2}\right)$ is above $45 \mathrm{mmHg}$, due to the disorder in the oxygenation and / or $\mathrm{CO}_{2}$ elimination functions of the respiratory system (1). Although for different pathophysiological reasons, many diseases can cause acute respiratory failure and these patients mostly require treatment and follow-up in intensive care units $(2,3)$.

With the increase of reactive oxygen species (ROS) such as hydroxyl radical, superoxide radical, and hydrogen peroxide formed during cellular metabolism and by the insufficiency of antioxidants responsible for their detoxification, deterioration in oxidative balance occurs, and oxidative stress develops. Increase in ROS causes cell damage or cell death by affecting macromolecules such as intracellular lipids, proteins, DNA and participates in the pathophysiology of many diseases $(4,5)$.

There are studies in the literature showing the role of oxidative stress in the pathogenesis of all infectious or inflammatory diseases that can cause respiratory failure, especially pneumonia and acute respiratory distress syndrome (ARDS) $(6,7)$. In these diseases, ROS released from high numbers of leukocytes damage the capillary endothelium and alveolar epithelium. Alveolar edema develops with this change in the alveolar epithelium, and alveolar collapse develops with the deterioration of the surfactant structure $(8,9)$.

Thiols are antioxidant agents among the mercaptan group used in the neutralization of reactive oxygen molecules in oxidative stress. Thiol synthesis can be performed in all eukaryotic living cells, and blood levels decrease as thiol groups are used for antioxidant purposes in the cell. The disulfide bonds formed by the interaction of thiols with oxidant molecules are transformed into thiol groups by reacting again with the proteins in the plasma thiol pool, and thus dynamic thiol / disulfide homeostasis is achieved. Dynamic thiol / disulfide homeostasis is only one of the many oxidant-antioxidant systems found in the human body and does not reflect the total antioxidant level of the body (10). There are studies showing the relationship of dynamic thiol / disulfide homeostasis with the pathogenesis of various diseases such as diabetes mellitus, cardiovascular diseases, cancer, rheumatoid arthritis, Parkinson's, Alzheimer's, and liver diseases (11-17).

Intensive care units are units where unstable patients that require intensive treatment and follow-up are accepted. Pathologies due to infection and / or inflammation such as pneumonia and ARDS constitute the majority of patients followed-up in intensive care units with the diagnosis of acute respiratory failure (18, 19). It was shown with various studies that oxidative stress plays a role in the etiopathogenesis of these diseases (20-22). If acute respiratory failure is diagnosed early, adequate oxygenation can be achieved with non-invasive supportive treatments. Invasive methods are needed to ensure adequate oxygenation in patients with severe acute respiratory failure. As a result of the accompanying nosocomial 
infections due to these invasive interventions, an increase in mortality may occur.

In this study, it was investigated that thiol / disulfide homeostasis is determined by an automatic, fast, cheap, and applicable method in patients who develop acute respiratory failure in the intensive care unit or who have been admitted to the intensive care unit due to acute respiratory failure, and that whether acute respiratory failure provides an advantage in early diagnosis. Hypothesis based on that lung damage due to oxidative stress can be detected earlier by deterioration of thiol / disulfide homeostasis was aroused. By this way it is aimed that the detection of oxidative stress biochemically can provide early diagnosis and rapid initiation of treatment.

\section{Materials and Methods}

This study was carried out prospectively from August 2018 till August 2019 in Intensive Care Department. Approval, with the decision numbered 2018 / 45, dated 01.26.2018, Gaziantep University Clinical Research Ethics Committee. Informed consent was obtained from the conscious patients and from the first-degree relatives of the unconscious patients.

\section{Patients and Control Groups}

A total of 98 patients, 58 males and 40 females, diagnosed with pneumonia and ARDS who were admitted to the intensive care unit due to acute respiratory failure and developed acute respiratory failure diagnosed with pneumonia, chronic obstructive pulmonary disease, pneumonia and asthma while in the intensive care unit, were defined as the patient group of the study. The control group consists of healthy individuals, who are at similar ages, in similar gender and do not have any known disease (59 males and 39 females,
98 individuals in total). Patients with $\mathrm{PaO}_{2}<60 \mathrm{mmHg}$ or $\mathrm{PaCO}_{2}>45 \mathrm{mmHg}$ were considered acute respiratory failure. Both the patient and control groups are older than 18 years of age and consent was obtained from the patient / patient relatives in order to participate in the study. Those with cardiovascular, cerebrovascular and rheumatic diseases, those with acutechronic kidney damage, those with malignancy and immunosuppression, diabetes mellitus patients, those who use antioxidant, lipid-lowering medication, anti-inflammatory drugs, or vitamin drugs, those who smoke, and pregnant women and those fewer than 18 years of age were excluded.

Blood samples and laboratory measurements

From patients who have been treated in the intensive care unit for any reason (excluding the exclusion criteria from the study) and from patients who develop acute respiratory failure $\left(\mathrm{PaO}_{2}<60 \mathrm{mmHg}\right.$ or $\mathrm{PaCO}_{2}>45 \mathrm{mmHg}$ ) while being treated in the service and from healthy volunteers, $6 \mathrm{ml}$ of venous blood was taken to biochemistry tubes with ethylenediaminetetraacetic acid (EDTA). The collected blood was centrifuged for 10 minutes at $1,800 \mathrm{rpm}$, the serum portion was separated and stored at $-80^{\circ} \mathrm{C}$ until analysis.

\section{Biochemical Evaluation}

For the thiol-disulfide balance (native thiol (-SH) - disulfide (-S-S-) change) in the patient and control groups, measurements were made using the spectrophotometric method (Rel Assay Diagnostics, Mega Tip, Turkey), which is the automatic measurement method found by Erel and Neşelioğlu (23). From these measurements, native thiol / total thiol, disulfide / total thiol, and disulfide / native 
thiol ratio were calculated. This measurement method is primarily based on the conversion of the disulfide bonds in the samples to functional thiol groups by sodium borohydride ( $\mathrm{NaBH} 4)$, then on the elimination by being consumed with formaldehyde to prevent the reduction of the unused reducing sodium borohydride, 5,5'- dithiobis- (2-nitrobenzoic) acid (DTNB). Thus, disulfide bonds that may be formed in the future are prevented. After reaction with DTNB, all thiol groups were identified, including the reduced and the "disulfide", "native thiol" and "total thiol" groups; sulfide amounts were calculated as native thiol/total thiol, disulfide/total thiol, disulfide/native thiol percentage ratios.

\section{Statistical Analyses}

For the statistical analysis of the data, SPSS (Statistical Package for Social Sciences) 22.0 version statistical program was used. The Shapiro-Wilk test was used to test whether the numerical variables were consistent with the normal distribution. The student's t-test was used to compare normally distributed variables in two groups, and the Mann-Whitney $U$ test was used to compare not normally distributed variables in two groups. $\mathrm{p}<0.05$ was considered significant.

\section{Results}

In this study, thiol and disulfide levels and thiol / disulfide ratios, which are a marker of total oxidant status, of intensive care patients with acute respiratory failure were compared with healthy volunteers. The method used in the study is new, applicable and valuable in terms of providing speed and convenience in patient management. In the study, 98 patients (58 males, 40 females), and 98 healthy volunteers (59 males and 39 females) were included. The mean ages of the patient and control groups were $63.15 \pm 16.36$ and $61.92 \pm 16.99$, respectively (Table 1$)$. The mean white blood cell (WBC) level of the patient group was $15.8\left(10^{3} / \mu\right)$, d-dimer level was $4.6(\mathrm{ug} / \mathrm{mL})$ and CRP levels were $160.36 \pm 16.81$ (mean \pm standard deviation). There was no significant change in other biochemical parameters.

Table 1: Demographic data of acute respiratory failure (patient) and control group.

\begin{tabular}{lccc}
\hline & $\begin{array}{c}\text { Patient Group } \\
(\mathbf{n = 9 8})\end{array}$ & $\begin{array}{c}\text { Control Group } \\
(\mathbf{n = 9 8 )}\end{array}$ & P \\
\hline Age & $63.15 \pm 16.36$ & $61.92 \pm 16.99$ & $>0.05$ \\
\hline Gender, n (\%) & & & $>0.05$ \\
Female & $40(40.8 \%)$ & $39(39.8 \%)$ & \\
Male & $58(59.2 \%)$ & $59(60.2 \%)$ & \\
\hline
\end{tabular}

There was no statistically significant difference between patient and control groups in terms of age and gender ( $p>$ $0.05)$.

Total thiol $(270 \pm 99.81)$, native thiol $(203.90 \pm 103.41)$, and disulfide $(33.10 \pm$ 12.42) levels in the patient group were found to be significantly lower than total thiol (423.62 \pm 70.3$)$, native thiol $(307.13$ \pm 57.73) and disulfide (58.24 \pm 27.21) levels in the control group $(\mathrm{p}=0.001$, Table 2).

There is no significant difference between the patient and control groups in terms of native thiol / total thiol, disulfide / total thiol, and disulfide/native thiol ratio ( $p$ > 
0.05 , Table 2).

Total thiol levels were found to be significantly lower in patients with acute respiratory failure than in healthy volunteers $(p=0.001)$ (Figure 1).

Native thiol levels were found to be significantly lower in patients with acute respiratory failure than in healthy volunteers $(p=0.001)$ (Figure 2).

Disulfide levels were found to be significantly lower in patients with acute respiratory failure than in healthy volunteers $(p=0.001)$ (Figure 3).

Table 2: Evaluation of thiol/disulfide parameters of patients with acute respiratory failure and control group.

\begin{tabular}{lccc}
\hline & $\begin{array}{c}\text { Patient Group } \\
(\mathbf{n = 9 8})\end{array}$ & $\begin{array}{c}\text { Control Group } \\
(\mathbf{n = 9 8})\end{array}$ & P \\
\hline Native thiol $(\boldsymbol{\mu m o l} / \mathbf{L})$ & $203.90 \pm 103.41$ & $307.13 \pm 57.73$ & 0.001 \\
\hline Total thiol $(\boldsymbol{\mu m o l} / \mathbf{L})$ & $270 \pm 99.81$ & $423.62 \pm 70.3$ & 0.001 \\
\hline Disulfide $(\boldsymbol{\mu m o l} / \mathbf{L})$ & $33.10 \pm 12.42$ & $58.24 \pm 27.21$ & 0.001 \\
\hline Disulfide/Native thiol $(\%)$ & 674.20 & 545.05 & $>0.05$ \\
\hline Disulfide/Total thiol $(\boldsymbol{\%})$ & 11.40 & 13.45 & $>0.05$ \\
\hline Native thiol/Total thiol $(\boldsymbol{\%})$ & $70.78 \pm 18.06$ & $72.88 \pm 11.28$ & $>0.05$ \\
\hline
\end{tabular}

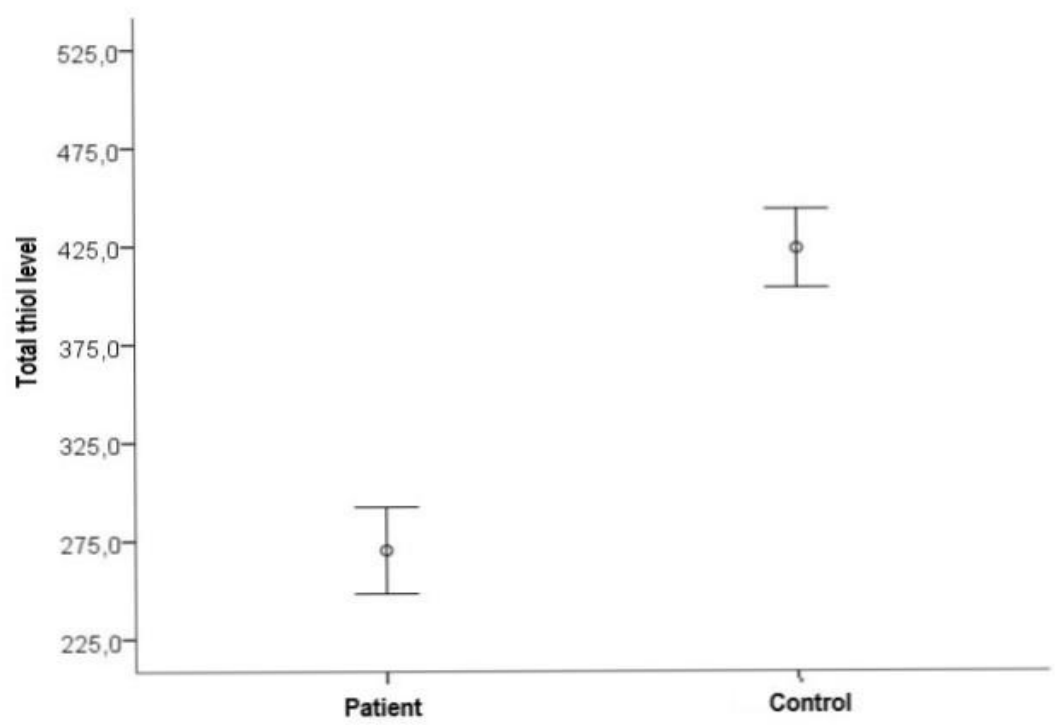

Figure 1: Total thiol levels of acute respiratory failure patients and control group. 


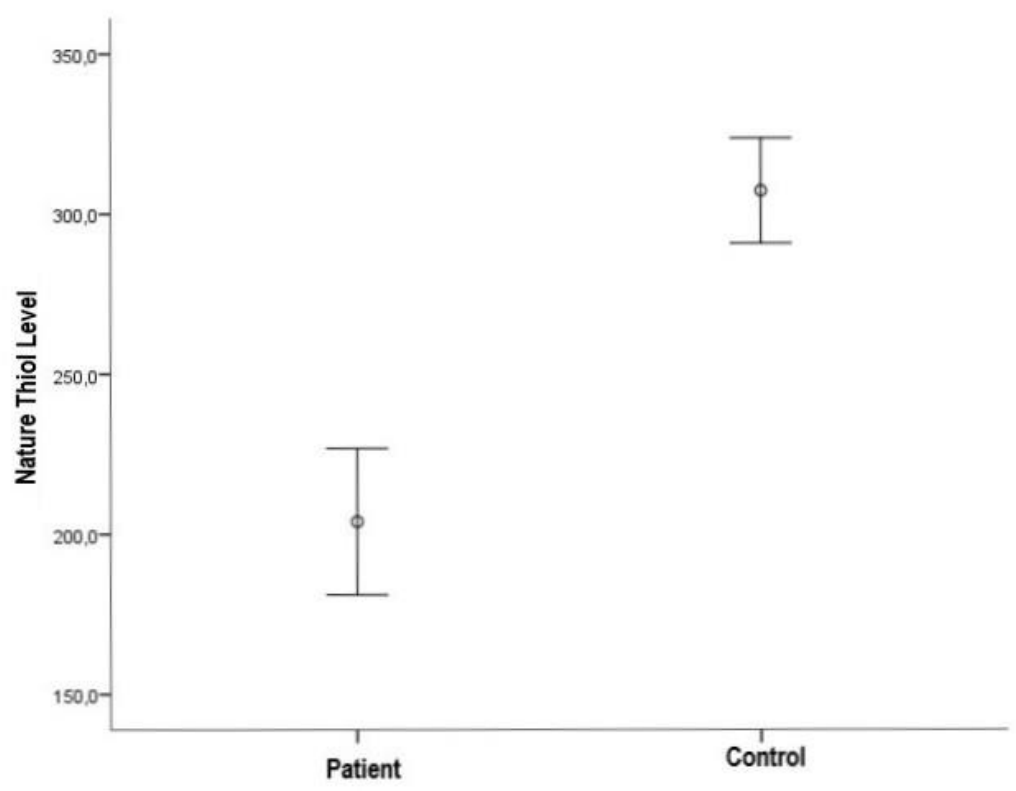

Figure 2: Native thiol levels of acute respiratory failure patients and control group.

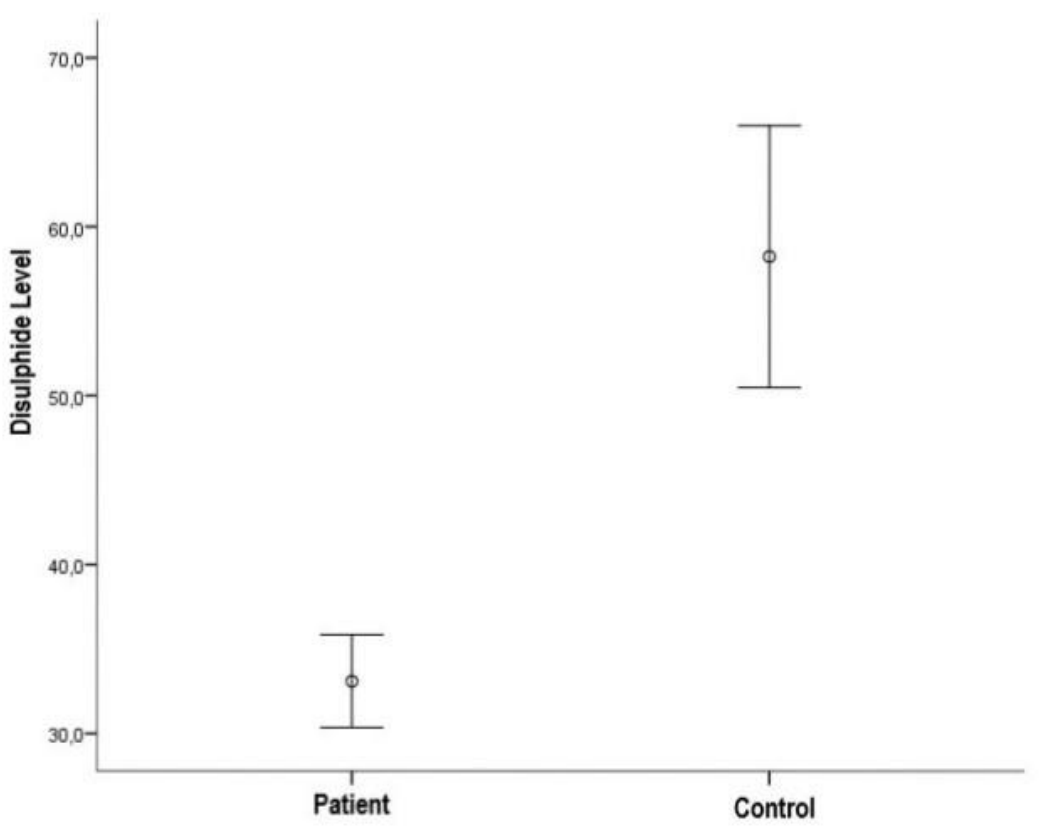

Figure 3: Disulfide levels of acute respiratory failure patients and control group.

There is no statistically significant difference between acute respiratory failure patients and healthy volunteers in terms of native thiol / total thiol ratio $(p>$ 0.05) (Figure 4).
There is no statistically significant difference between acute respiratory failure patients and healthy volunteers in terms of disulfide / total thiol ratio ( $p>$ 0.05) (Figure 5). 


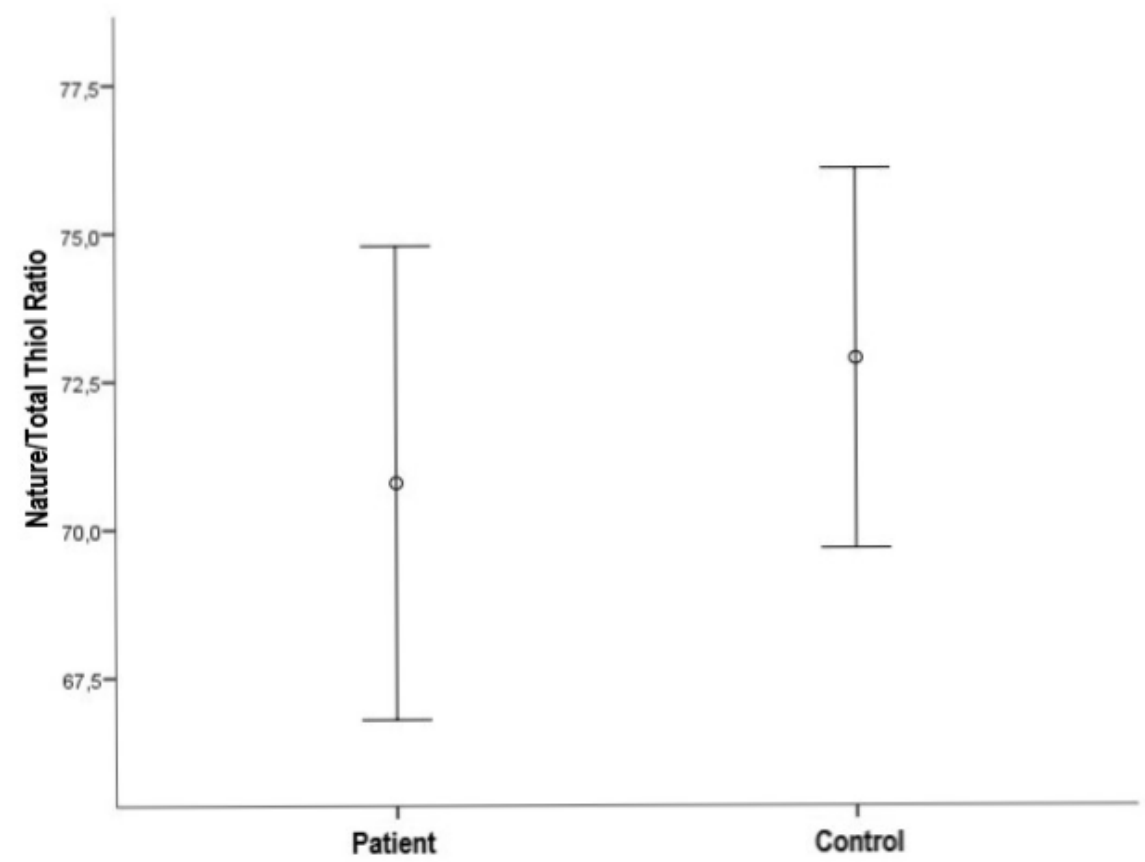

Figure 4: Native thiol/total thiol ratio of patients with acute respiratory failure and control group.

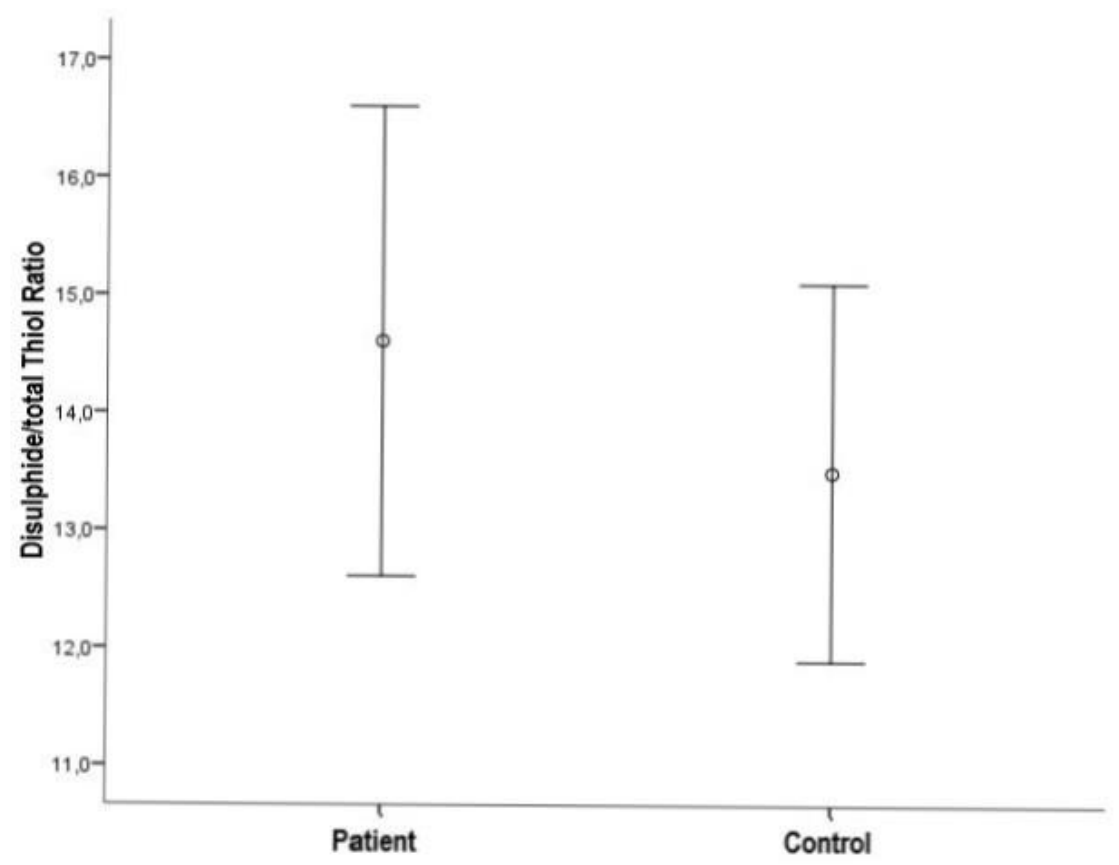

Figure 5: Disulfide/total thiol ratio of acute respiratory failure patients and control group.

There is no statistically significant difference between acute respiratory failure patients and healthy volunteers in terms of disulfide/native thiol ratio $(p>$ 0.05) (Figure 6). 


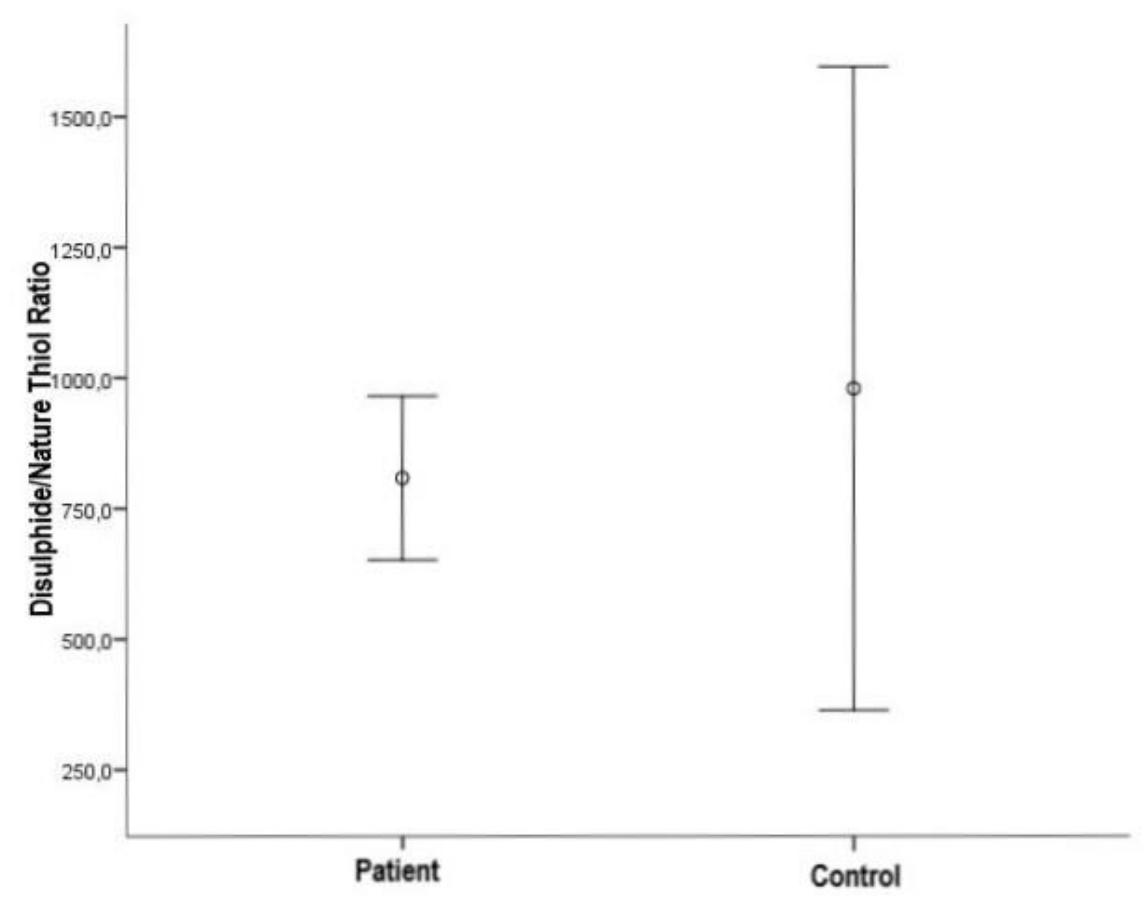

Figure 6: Disulfide/native thiol ratio of acute respiratory failure patients and control group.

\section{Discussion}

In this study, thiol and disulfide blood levels and thiol / disulfide ratios, which are indicators of total oxidant status in intensive care patients with respiratory failure, were compared with healthy volunteers with a new, feasible method that will provide speed and convenience in patient management. Total thiol, native thiol, and disulfide levels were found to be statistically significantly lower in the patient group with respiratory failure compared to the control group. There was no significant difference in the ratio of native thiol / total thiol, disulfide / total thiol, and disulfide / native thiol. As far as we know, our study is the first study in the literature in terms of providing the diagnosis and follow-up of dynamic thioldisulfide balance using an automatic, fast, and inexpensive method and initiating treatment in patients with acute respiratory failure requiring intensive care support treatment.
Oxidative stress is the deterioration of the prooxidant-antioxidant balance in the body and tissues. The formation of ROS known as prooxidants is a natural result of normal aerobic life. The existence and development of cells in oxygen-containing environments is not possible without powerful antioxidant enzymes and nonenzyme antioxidant systems. In aerobic life, constantly occurring prooxidants must be regularly absorbed by antioxidants and balanced by consumption. Otherwise, oxidative damage will occur and pathophysiological events may develop with its accumulation.

Thiols are antioxidant agents that consist of sulfur and carbon atoms and are used for the neutralization of ROS in oxidative stress situations. The disulfide bonds formed by the interaction of thiol groups with oxidant molecules interact with the proteins in the plasma thiol pool and are transformed back into the thiol group and dynamic thiol / disulfide homeostasis is 
achieved (24-26). Dynamic thiol / disulfide homeostasis is associated with the pathogenesis of malignancies, cardiovascular, neurodegenerative, and inflammatory diseases (23).

In a healthy lung, the continuation of normal physiological functions is provided by the continuation of the balance between oxidants and antioxidants in the airway and extracellular areas. The lung is a tissue that can easily be damaged by ROS, which has high oxygen content. The increase of ROS stimulates mucus secretion, inactivates antiproteases and controls cell proliferation or apoptosis.

In respiratory tract infections, the entry and activation of phagocytes to the lower respiratory tract provides protection against ROS and lysosomal enzymes and microorganisms. Therefore, systemic ROS concentration may increase in respiratory tract infections (27). High ROS levels were seen to be associated with inflammatory reactions such as transcriptional modification, signal transduction, or gene expression of pro-inflammatory mediators (28). In respiratory tract infections, ROS released from polymorphonuclear leukocytes (PMNL) and macrophages cause tissue damage in the respiratory tract, and lung cells release inflammatory mediators and cytokines / chemokines to their environment in response to oxidative stress (27).

Smoking, acute exacerbations of chronic obstructive pulmonary disease (COPD), pneumonia, and asthma are clinical pictures in which oxidant / antioxidant balance deteriorated and oxidative stress is present $(29,30)$. Thiol levels, which are an indicator of oxidant / antioxidant imbalance in these diseases, have been shown to change with the severity of the disease and also with the treatment response.

Patients who develop acute respiratory failure and require intensive care are usually diagnosed with sepsis, pneumonia or acute exacerbation of COPD. The patients that included in this study were those who were being treated in the intensive care unit with a diagnosis of pneumonia or who were diagnosed with pneumonia that needed intensive care due to the development of acute respiratory failure.

In two different studies conducted in adult patients hospitalized with the diagnosis of community-acquired pneumonia and in the pediatric patient group, total thiol and native thiol levels have been found to be significantly lower in patients compared to the control group $(27,31)$. These findings are consistent with the findings we obtained in our study. However, in the study conducted by Parlak et al., they have shown that the disulfide/total thiol ratio was significantly higher in the patient group compared to the control group (27). They have interpreted this highness as the timely participation of some antioxidant mechanisms in the oxidative stress process and the ability to transform disulfide bonds back to thiol groups, thus keeping the thiol/disulfide homeostasis in balance. In our study, disulfide/total thiol ratios were not different in the patient and control groups. This can be interpreted as that antioxidant mechanisms could not participate in the oxidative stress process in time, as our study group consisted of patients with acute respiratory failure. In another study conducted by Temel et al., consistent with our findings, it has been found that native thiol, total thiol, and disulfide levels to be lower in children with community-acquired pneumonia than in the control group (31). However, unlike 
this study, they have found the ratio of native thiol / total thiol to be lower, and the disulfide / native thiol and disulfide/total thiol ratio to be higher than the control group. In this study, the researchers have interpreted that a high amount of ROS was released from the increased leukocytes in the patient group and thus a high disulfide level was formed. Similarly, in a study conducted in patients with urinary stones, there has been a significant decrease in native thiol and total thiol levels compared to controls, while disulfide levels have been found to be significantly higher (32). In this approach, if considered, the results can be expected in infectious diseases with leukocytosis. The leukocyte levels of the patients participating in our study were found to be above normal limits. Therefore, the lower levels of total thiol, native thiol and disulfide compared to the controls can be explained by the fact that ROS released from a high number of leukocytes oxidize thiols and decrease their levels.

The results obtained from studies conducted in patients with COPD and asthma with inflammation in the etiopathogenesis as well as causes related to infection are also consistent with our results $(28,30,33,34)$. Babaoğlu et al. have emphasized the importance of oxidant / antioxidant balance in tissues and blood in obstructive pulmonary diseases and investigated the thiol / disulfide balance in patients (28). Thiol / disulfide homeostasis parameters have been found to be similar in all three patient groups (patients with asthma, COPD, and COPD overlap syndrome) who were taken as obstructive pulmonary disease. This result shows that all three diseases have similar pathophysiology but different clinic findings (28). Gündüzöz et al. have shown that firefighters exposed to chronic xenobiotics had abnormal respiratory function tests and chest radiographs, and significantly higher levels of disulfide and disulfide / native thiol. They have emphasized that oxidative stress may play an important role in the pathogenesis of lung injury in these individuals and that early detection of oxidative stress is important (35).

Effective use of intensive care beds is extremely important for the provision of hospital services and patient health. Patients who rank in priority and admitted to the intensive care units are unstable patients requiring intensive treatment and observation. Patients who developed acute respiratory failure constitute the priority patient group for intensive care units. The majority of patients with acute respiratory failure admitted to intensive care units consist of patients with sepsis, pneumonia, and ARDS. These patients, instead of the fact that they can regain their health with early diagnosis and treatment of respiratory distress, they have to fight with nosocomial infections rather than hospitalization reasons, due to the prolongation of stay in intensive care units due to delays in diagnosis and treatment, and the necessity of more invasive methods, and even die due to infection. Therefore, early diagnosis of respiratory failure and early intervention may eliminate the indication of hospitalization in the intensive care unit and shorten the stay in the intensive care unit of the patient who is already inpatient in the intensive care unit.

In this study, it was shown that native thiol, total thiol, and disulfide levels were significantly decreased in intensive care patients with acute respiratory failure. Determining the levels of thiol groups may be important in the prognosis, clinical 
management, diagnosis and follow-up of diseases in which ROS are involved in the pathogenesis, increasing the survival of patients and shortening the duration of stay in intensive care. Our results are consistent with previous studies conducted in patients in whom oxidative stress plays a role in the pathogenesis of COPD, asthma and pneumonia.

The degree of respiratory failure and dynamic thiol-disulfide homeostasis could not be evaluated together in patients with acute respiratory failure. Since the patients we included in the study are patients who are being followed up in the intensive care unit and are unstable in terms of pulmonary functions, oxygen saturation and partial oxygen pressure values in the blood may vary until the general condition of the patient is stable. As a large number of blood samples can be taken during the day and this evaluation can be made, it is practically mandatory.

As a result, we predict that the fast, easy and accessible method we use to measure native thiol, total thiol, and disulfide levels in patients with acute respiratory failure may be a suitable biomarker in terms of diagnosing respiratory failure and providing ease of patient follow-up.

\section{Conflict of Interest}

The authors declare that they have no conflict of interest.

\section{Acknowledgment}

No institution has given financial support to the study. All researchers contributed equally to the study.

\section{References}

1. Yarkın T. Solunum Yetmezliği:
Fizyopatoloji ve Klinik Yaklaşım. Toraks Dergisi.2000;2:76-84.

2. Frutos-Vivar F, Nin N, Esteban A. Epidemiology of acute lung injury and acute respiratory distress syndrome.Curr Opin Crit Care. 2004;10(1):1-6.

3. Esteban A, Anzueto A, Frutos F, et al. Mechanical Ventilation International Study Group. Characteristics and outcomes in adult patients receiving mechanical ventilation: a 28-day international study. JAMA. 2002;16:287(3):345355.

4. Becatti M, Taddei N, Fiorillo C. Oxidative stress management during non-invasive ventilation in acute respiratory failure. Intern Emerg Med. 2018;13:141-142.

5. Özcan O, Erdal H, Çakırca G, et al. Oxidative stress and its impacts on intracellular lipids, proteins and DNA. Journal of Clinical and Experimental Investigations. 2015;6(3):331-336.

6. Gutteridge JMC, Quinlan GJ, Mumby S, et al. Primary plasma antioxidants in adult respiratory distress syndrome patients: changes in ironoxidizing, iron-binding and free radical-scavenging proteins. J. Lab. Clin. Med. 1994;124:263-273.

7. Hecker L. Mechanisms and consequences of oxidative stress in lung disease: therapeutic implications for an aging populace. Am J Physiol Lung Cell Mol Physiol. 2018;314(4):642-653.

8. Quinlan GJ. Lamb NJ, Tilley R, et al. Plasma hypoxanthine levels in ARDS: implications for oxidative stress, morbidity, and mortality. American Journal of Respiratory and Critical Care Medicine. 1997;155(2):479-484.

9. Gürsel G. Akut Solunum Sikıntıs1 Sendromu (ARDS). Yoğun Bakım Dergisi. 2002;2(2):96-107.

10. Sen CK, Packer L. Thiol homeostasis and supplements in physical exercise. Am J Clin Nutr. 2000;72(2 Suppl.):653-669.

11. Matteucci E, Giampietro O. Thiol signalling network with an eye to diabetes. Molecules 2010;15(12):8890-903.

12. Go YM, Jones DP. Cysteine/cystine redox signaling in cardiovascular disease. Free Radic Biol Med. 2011;15:50(4):495509.

13. Prabhu A, Sarcar B, Kahali S, et al. Cysteine catabolism: a novel metabolic pathway contributing to glioblastoma growth. Cancer Res 2014;74(3):787-96.

14. Tetik S, Ahmad S, Alturfan AA, et al. Determination of oxidant stress in plasma of 
rheumatoid arthritis and primary osteoarthritis patients. Indian J Biochem Biophys. 2010;47(6):353-358.

15. Calabrese V, Lodi R, Tonon C, et al. Oxidative stress, mitochondrial dysfunction and cellular stress response in Friedreich's ataxia. J Neurol Sci. 2005;15:233(1-2):145-62.

16. Steele ML, Fuller S, Maczurek AE, et al. Chronic inflammation alters production and release of glutathione and related thiols in human U373 astroglial cells. Cell Mol Neurobiol. 2013;33(1):1930 .

17. Kuo LM, Kuo CY, Lin CY, et al. Intracellular glutathione depletion by oridonin leads to apoptosis in hepatic stellate cells. .Molecules. 2014;18:19(3):3327-3344.

18. Frutos-Vivar F, Nin N, Esteban A. Epidemiology of acute lung injury and acute respiratory distress syndrome. Curr Opin Crit Care. 2004;10(1):1-6.

19. Esteban A, Anzueto A, Frutos F, et al. Mechanical Ventilation International Study Group. Characteristics and outcomes in adult patients receiving mechanical ventilation: a 28-day international study. JAMA. 2002;16:287(3):345355.

20. Quinlan GJ. Lamb NJ, Tilley R, et al. Plasma hypoxanthine levels in ARDS: implications for oxidative stress, morbidity, and mortality. American Journal of Respiratory and Critical Care Medicine. 1997;155(2):479-484.

21. Baldwin S, Grum C, Boxer L, et al. Oxidant activity in expired breath of patients with adult respiratory distress syndrome. The Lancet. 1986;327(8471):11-14.

22. Lenz AG, Jorens PG, Meyer B, et al. Oxidatively modified proteins in bronchoalveoler lavage fluid of patients with ARDS and patients atrisk for ARDS. Eur Respir J 1999;13: 169-174.

23. Erel O, Neselioglu S. A novel and automated assay for thiol/disulfide homeostasis. Clinical Biochem. 2014;47(18):326-332.

24. Sen CK, Packer L. Thiol homeostasis and supplements in physical exercise. Am J Clin Nutr. 2000;72(2 Suppl.):653-669.

25. Turell L, Radi R, Alvarez B. The thiol pool in human plasma: The central contribution of albumin to redox processes. Free Radic. Biol. Med. 2013; 65: 244-253.

26. Jones DP, Liang Y. Measuring the Poise of Thiol/Disulfide Couples in vivo. Free Radic Biol Med. 2009; 47(10): 1329-1338.

27. Parlak ES, Alisik M, Hezer $\mathrm{H}$, et al. Evaluation of dynamic thiol/disulfide redox state in community-acquired pneumonia. Saudi Med J 2018;39(5): 495-499.

28. Babaoglu E, Kilıc H, Hezer H, et al. European Review for Medical and Pharmacological Sciences. Comparison of thiol/disulfide homeostasis parameters in patients with COPD, asthma and ACOS. 2016;20:1537-1543.

29. Ahmad A, Shameem M, Husain Q. Altered oxidant-antioxidant levels in the disease prognosis of chronic obstructive pulmonary disease. Int $\mathbf{J}$ Tuberc Lung Dis. 2013;17(8):1104-1109.

30. Dilek F, Ozkaya E, Kocyigit A, et al. Plasma total thiol pool in children with asthma: Modulation during montelukast monotherapy. International Journal of Immunopathology and Pharmacology. 2016;29(1):84-89.

31. Temel MT, Demiryurek S, Temel L, et al. Dynamic thiol/disulfide homeostasis in children with community acquired Pneumonia. Pediatr Int. 2019;61(3):252-257

32. Sonmez MG, Kozanhan B, Deniz CD, et al. Dynamic thiol/disulfide homeostasis as a novel indicator of oxidative stress in patients with urolithiasis. Investig Clin Urol. 2019;60:258-266.

33. Raut AM, Suryakar AN, Mhassekar D. A study of oxidative stress, thiol proteins and role of vitamin $\mathrm{E}$ supplementation in chronic obstructive pulmonary disease (COPD). Al Ameen J Med Sci 2013;6:134-137.

34. Erden ES, Motor S, Ustun I, et al. Investigation of Bisphenol $\mathrm{A}$ as an endocrine disruptor, total thiol, malondialdehyde, and Creactive protein levels in chronic obstructive pulmonary disease. Eur Rev Med Pharmacol Sci. 2014;18(22):3477-3483.

35. Gündüzöz M, Birgin İS, Tutkun L, et al. A new potential biomarker in early diagnosis of firefighter lung function impairment: dynamic thiol/disulfide homeostasis. Cent Eur J Public Health. 2018;26(3):190-194. 